\title{
Relation Between Cancer and Vasospastic Angina
}

Tadahiro Matsumoto $\cdot$ Yuichi Saito (1) - Kan Saito $\cdot$ Kazuya Tateishi

Ken Kato $\cdot$ Hideki Kitahara $\cdot$ Yoshio Kobayashi

Received: May 27, 2021 / Accepted: June 29, 2021 / Published online: July 9, 2021

(C) The Author(s) 2021

\section{ABSTRACT}

Introduction: Patients with cancer have an increased risk of cardiovascular disease including ischemic heart disease and vice versa. Anticancer drugs and radiotherapy are known to contribute to endothelial injury and vasospasm. However, the relations between vasospastic angina (VSA) and cancer or its treatment are poorly investigated.

Methods: A total of 786 patients underwent intracoronary acetylcholine $(\mathrm{ACh})$ provocation tests to diagnose VSA. The positive ACh provocation test was defined as angiographic coronary artery spasm accompanied by chest pain and/or ischemic electrocardiographic changes. Patients were divided into active cancer, a history of cancer, and no cancer according to the status of malignancy. The impact of types of cancer, anticancer drugs, and radiotherapy on VSA was evaluated.

Results: Of 786 patients, 38 (4.8\%) and 84 $(10.7 \%)$ had active cancer and a history of

Supplementary Information The online version contains supplementary material available at https:// doi.org/10.1007/s12325-021-01854-z.

T. Matsumoto · Y. Saito $(\bowtie) \cdot$ K. Saito · K. Tateishi .

K. Kato $\cdot$ H. Kitahara · Y. Kobayashi

Department of Cardiovascular Medicine, Chiba

University Graduate School of Medicine, Chiba,

Japan

e-mail: saitoyuichi1984@gmail.com cancer, respectively, and 401 (51.0\%) were diagnosed as VSA. There was no significant difference in rates of positive ACh test among patients with active cancer, a history of cancer, and no cancer $(39.5 \%$ vs. $57.1 \%$ vs. $50.9 \%$, $p=0.20$ ). Types of cancer and cancer treatment also had no impact on positive ACh provocation test.

Conclusions: In this cross-sectional observational study, we did not find an association of active and a history of cancer with the diagnosis of VSA. Anticancer treatment including chemotherapy and radiotherapy was not significantly associated with positive ACh provocation test.

Keywords: Vasospastic angina; Cancer; Anticancer drug; Acetylcholine provocation 


\section{Key Summary Points}

Why carry out this study?

Cancer and its treatment are considered to contribute to endothelial injury and vasospasm

However, relations between vasospastic angina (VSA) and cancer are poorly defined

\section{What was learned from the study?}

Cancer status and type had no impact on positive acetylcholine provocation test

Anticancer treatment was not significantly associated with VSA

Further studies are needed to elucidate the direct association of cancer with VSA

\section{INTRODUCTION}

Vasospastic angina (VSA) is an important cardiac disorder that is associated with the deterioration of quality of life and can induce myocardial infarction and sudden cardiac death [1]. Endothelial dysfunction with deficiency of nitric oxide plays a key role in the mechanism of VSA [2], as well as inflammation, autonomic nerve system, and rho-kinase activity $[3,4]$.

Heart disease and cancer are the leading causes of death worldwide especially in developed countries [5], and patients with cancer have an increased risk for cardiovascular events and mortality from shared lifestyles, risk factors, and underlying mechanisms such as inflammation, endothelial dysfunction, and oxidative stress [6-11]. Toxicities of cancer treatment, including chemotherapy and radiotherapy, also contribute to endothelial injury and vasospasm, as described in the European Society of Cardiology position paper [12]. In this context, a significant association of anticancer drugs with VSA has been indicated. A case report demonstrated coronary spasm induced by 5-fluorouracil (5-FU) [13], and sorafenib, an inhibitor of the vascular endothelial growth factor (VEGF) receptors, was related to VSA with the potential mechanism of upregulation of rho-kinase activity $[14,15]$. Thus, the presence or a history of cancer and chemotherapy and/or radiotherapy may be associated with coronary vasospasm. The aim of this study was to investigate the impact of cancer and its treatment on VSA.

\section{METHODS}

\section{Study Population}

Between April 2012 and June 2020, a total of 809 consecutive patients underwent intracoronary acetylcholine (ACh) provocation test to diagnose VSA at Chiba University Hospital. Patients who had experienced out-of-hospital cardiac arrest $(n=23)$ were excluded. Thus, 786 patients were retrospectively included in the present study. Written informed consent for examination was obtained from all patients, and informed consent for the present study was obtained in the form of opt-out (no patients chose to opt out of participating in this study). This study was conducted in accordance with the Declaration of Helsinki, and the ethical committee of Chiba University Graduate School of Medicine approved the present study (Approval No. 3983).

\section{Cancer Definitions}

Patients were divided into three groups according to the status of malignancy: active cancer, a history of cancer, and no cancer. Patients who were planned for surgery for cancer, were receiving anticancer $\operatorname{drug}(\mathrm{s})$ and/or radiotherapy and had recurrent, metastatic, or inoperable cancer were defined as having active cancer. Cancer type included oral cavity and pharynx, thyroid, lung, breast, esophagus, stomach, small intestine, colon and rectum, hepatobiliary pancreas, kidney, bladder, ovary, prostate, uterus, skin, and blood. Anticancer treatment included chemotherapy with anticancer drugs 
and radiotherapy. Anticancer drugs were categorized as follows: molecularly targeted drug (bevacizumab, sunitinib, and dasatinib), cytotoxic anticancer drug (cisplatin, docetaxel, fluorouracil, and tegafur/gimeracil/oteracil), and hormonal anticancer drug (anastrozole, leuprorelin, letrozole, and vicartamide).

\section{Intracoronary Acetylcholine Provocation Tests}

Intracoronary $\mathrm{ACh}$ provocation tests were performed to diagnose VSA based on the guidelines $[16,17]$, as previously reported $[4,18-23]$. In brief, all vasodilators including calcium channel blockers and long-acting nitrates were discontinued at least $48 \mathrm{~h}$ before the examination in elective cases, except for sublingual nitroglycerin as needed. The radial artery and brachial vein were mainly used as approach sites [24]. After control angiography, a temporary pacing electrode was inserted in the right ventricle. Intracoronary ACh was administered in incremental doses of 20, 50, and $100 \mu \mathrm{g}$ into the left coronary artery initially, and 20 and $50 \mu \mathrm{g}$ into the right coronary artery subsequently, over a period of $20 \mathrm{~s}$. One minute after the start of each injection, coronary angiography was performed to evaluate coronary vasospasm. After ACh provocation testing, 1-2 mg of isosorbide dinitrate was administered into the right and left coronary arteries, and coronary angiography was performed. Obstructive epicardial coronary artery disease (CAD) was defined as $\geq 50 \%$ stenosis on coronary angiography after administration of intracoronary isosorbide dinitrate. The positive ACh provocation test was defined as angiographic coronary artery spasm, a total or subtotal occlusion by the ACh administration, accompanied by chest pain and/or ischemic electrocardiographic changes. It was evaluated by two experienced cardiologists who were blinded to patients' clinical characteristics.

\section{Endpoint and Statistical Analysis}

The primary endpoint of the present study was positive ACh provocation test in patients with different cancer statuses. Relations between VSA and cancer type or treatment were also evaluated. All statistical analyses were conducted using JMP Pro 15.0.0 (SAS Institute, Cary, NC, USA). Data are expressed as median [interquartile range] or frequency (\%). Continuous variables were compared using Kruskal-Wallis and Mann-Whitney $U$ tests. Normal distribution was tested using Shapiro-Wilk test. Categorical variables were compared with Fisher's exact test. Separate logistic regression analyses were performed to identify univariable predictors of positive ACh provocation test. The associated variables in univariable analyses $(p<0.20)$ and age, sex, and active cancer (irrespective of $p$ value) were included in the model of multivariable logistic regression analysis. Because anticancer drug was highly correlated with active cancer, the two factors were not included in the multivariable model simultaneously. A value of $p<0.05$ was considered statistically significant.

\section{RESULTS}

Of 786 patients, 38 (4.8\%) and 84 (10.7\%) had active cancer and a history of cancer, respectively, and 401 (51.0\%) were diagnosed as VSA with positive ACh provocation test. Patients with cancer were older and had lower body mass index among the three groups (Table 1). In terms of ACh provocation test findings, the number of provoked angiographic coronary artery spasms was significantly fewer in patients with active cancer, while the rate of positive ACh provocation test was not different among the three groups (Table 2). Figure 1 shows relations between cancer type and VSA in patients with active and a history of cancer. The rates of positive ACh provocation test ranged from 33\% to $100 \%$ among types of cancer with no significant differences. When focusing on only patients with active cancer, no significant differences were observed (Fig. 2). Among patients with active cancer, 16 were receiving anticancer drug (4 molecularly targeted, 4 cytotoxic, and 9 hormonal drugs) and 4 underwent ongoing radiotherapy. Cancer treatment also had no impact on positive ACh provocation test (Fig. 3). Multivariable analysis identified current 
Table 1 Baseline characteristics

\begin{tabular}{|c|c|c|c|c|}
\hline Variable & Active cancer $(n=38)$ & History of cancer $(n=84)$ & No cancer $(n=664)$ & $p$ value \\
\hline Age (years) & $74.0[66.0,78.3]$ & $72.0[65.3,78.0]$ & $65.0[54.0,72.0]$ & $<0.001$ \\
\hline Men & $22(57.9 \%)$ & $53(63.1 \%)$ & $329(49.6 \%)$ & 0.049 \\
\hline Body mass index $\left(\mathrm{kg} / \mathrm{m}^{2}\right)$ & $22.1[21.3,25.7]$ & $23.3[20.3,26.2]$ & $23.4[21.3,25.7]$ & 0.09 \\
\hline Hypertension & $25(65.8 \%)$ & $52(61.9 \%)$ & $387(58.3 \%)$ & 0.58 \\
\hline Diabetes mellitus & $10(26.3 \%)$ & $19(22.6 \%)$ & $117(17.6 \%)$ & 0.21 \\
\hline Dyslipidemia & $24(63.2 \%)$ & $51(60.7 \%)$ & $428(64.5 \%)$ & 0.77 \\
\hline Current smoker & $6(15.8 \%)$ & $15(17.9 \%)$ & $114(17.2 \%)$ & 0.97 \\
\hline Prior myocardial infarction & $4(10.5 \%)$ & $6(7.2 \%)$ & $53(8.1 \%)$ & 0.78 \\
\hline ACS presentation & $9(23.7 \%)$ & $18(21.4 \%)$ & $129(19.4 \%)$ & 0.69 \\
\hline \multicolumn{5}{|l|}{ Clinical presentation } \\
\hline Rest angina & $26(68.4 \%)$ & $56(66.7 \%)$ & $485(73.0 \%)$ & 0.39 \\
\hline Effort angina & $2(5.3 \%)$ & $4(4.8 \%)$ & $14(2.1 \%)$ & 0.13 \\
\hline Rest and effort angina & $7(18.4 \%)$ & $20(23.8 \%)$ & $145(21.8 \%)$ & 0.82 \\
\hline No angina & $3(7.9 \%)$ & $4(4.8 \%)$ & $20(3.0 \%)$ & 0.16 \\
\hline \multicolumn{5}{|l|}{ Medical treatment } \\
\hline Calcium channel blocker & $17(44.7 \%)$ & $37(44.1 \%)$ & $301(45.3 \%)$ & 0.99 \\
\hline Long-acting nitrate & $12(31.6 \%)$ & $18(21.4 \%)$ & $110(16.6 \%)$ & 0.048 \\
\hline Antiplatelet & $12(31.6 \%)$ & $29(34.5 \%)$ & $194(29.2 \%)$ & 0.55 \\
\hline Statin & $10(26.3 \%)$ & $30(35.7 \%)$ & $255(38.4 \%)$ & 0.32 \\
\hline ACE-I or ARB & $18(47.4 \%)$ & $28(33.3 \%)$ & $218(32.8 \%)$ & 0.19 \\
\hline$\beta$-blocker & $9(23.7 \%)$ & $13(15.5 \%)$ & 97 (14.6\%) & 0.30 \\
\hline
\end{tabular}

$A C S$ acute coronary syndrome, $A C E-I$ angiotensin converting enzyme inhibitor, $A C h$ acetylcholine, $A R B$ angiotensin II receptor blocker

smoking and obstructive epicardial $\mathrm{CAD}$ as factors associated with VSA, but cancer-related variables were not associated (Table 3).

\section{DISCUSSION}

The present study included 5\% and $11 \%$ of patients having active and a history of cancer in a cohort of patients with suspected VSA undergoing intracoronary $\mathrm{ACh}$ provocation test. Overall, 51\% was diagnosed as VSA, but the status of cancer was significantly not associated with positive ACh provocation test. In addition, we did not find impact of type of cancer and anticancer treatment including chemotherapy and radiotherapy on positive ACh test. Multivariable analysis reinforced the findings. To the best of our knowledge, this is the first study investigating relations between VSA and cancer or its treatment. 
Table 2 ACh provocation test findings

\begin{tabular}{lllll}
\hline Variable & Active cancer $(\boldsymbol{n}=\mathbf{3 8})$ & History of cancer $(\boldsymbol{n}=\mathbf{8 4})$ & No cancer $(\boldsymbol{n}=\mathbf{6 6 4})$ & $\boldsymbol{p}$ value \\
\hline Positive ACh provocation test & $15(39.5 \%)$ & $48(57.1 \%)$ & $338(50.9 \%)$ & 0.20 \\
Signs of ischemia & & & \\
ECG change & $13(34.2 \%)$ & $37(44.1 \%)$ & $278(41.9 \%)$ & 0.59 \\
Chest pain & $17(44.7 \%)$ & $47(56.0 \%)$ & $368(55.4 \%)$ & 0.44 \\
Number of spasm vessels & $0[0,1]$ & $1[0,2]$ & $1[0,2]$ & 0.01 \\
Multivessel spasm & $8(21.1 \%)$ & $31(36.9 \%)$ & $179(27.0 \%)$ & 0.11 \\
Obstructive epicardial CAD & $14(36.8 \%)$ & $27(32.1 \%)$ & $173(26.1 \%)$ & 0.18 \\
\hline
\end{tabular}

$A C h$ acetylcholine, $C A D$ coronary artery disease, $E C G$ electrocardiogram

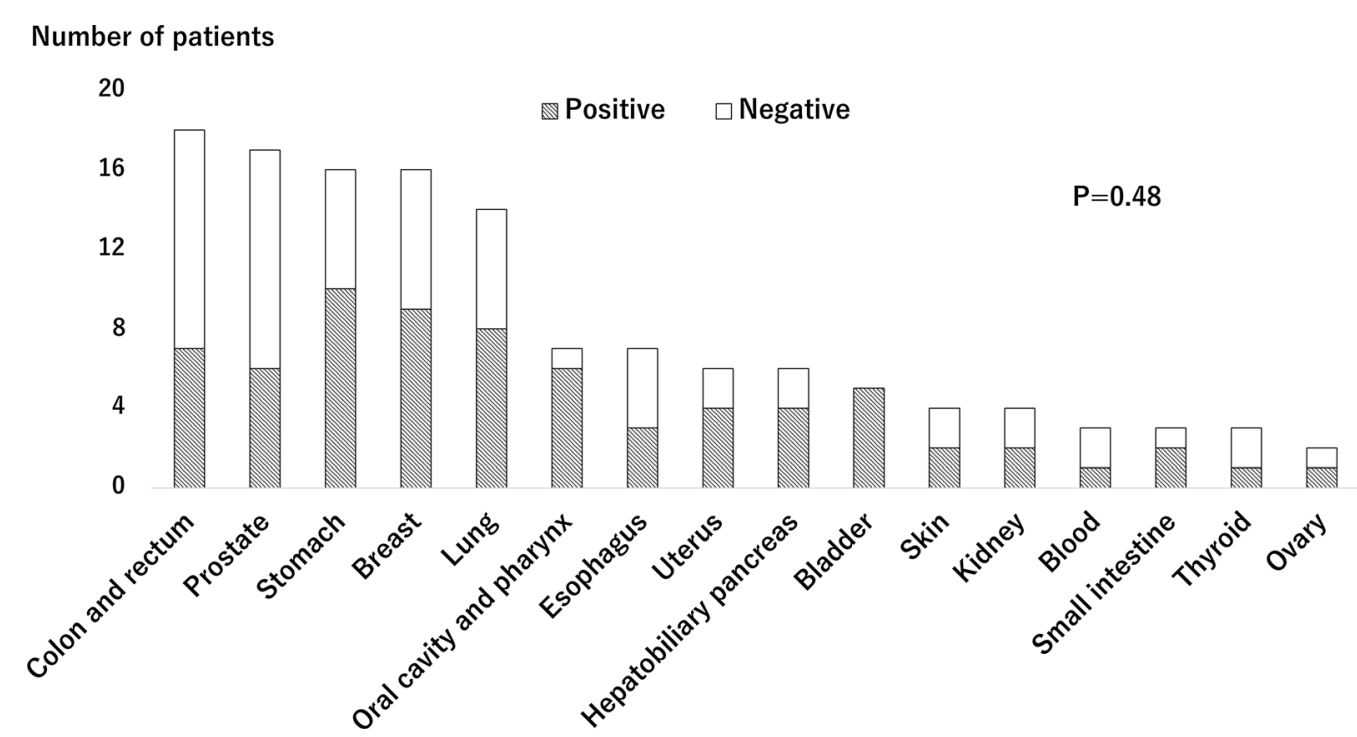

Fig. 1 Positive rates of acetylcholine provocation test across types of cancer in patients with active and a history of cancer

\section{Cancer and Ischemic Heart Disease}

Patients with cancer have an increased risk of cardiovascular disease including ischemic heart disease and vice versa [8-11]. A large-scale national registry including $>6.5$ million participants showed that in patients with acute myocardial infarction, $2.8 \%$ had active cancer and $6.2 \%$ had a history of cancer [25]. In this registry, patients with active cancer had higher mortality as expected, but they were also at a higher risk for major adverse cardiovascular and cerebrovascular events compared with patients with no cancer [25]. VSA, a part of ischemic heart disease, reportedly accounts for approximately $40 \%$ of all angina in East Asian populations [16]. Given that patients with cancer and ischemic heart disease including VSA have shared lifestyle and risk factors (e.g., smoking and metabolic syndrome) and underlying mechanisms (e.g., inflammation, endothelial dysfunction, and oxidative stress) $[9,10]$, it 


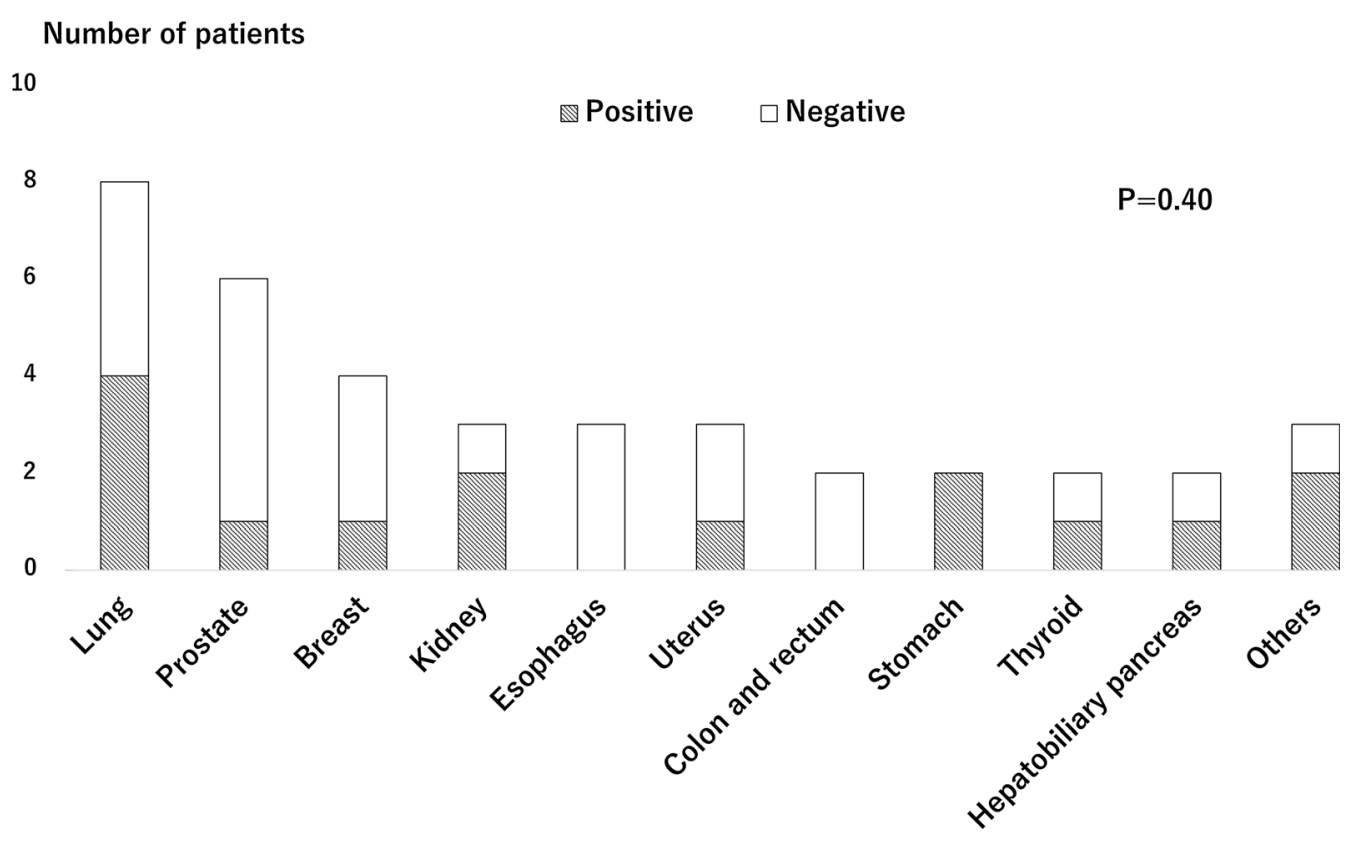

Fig. 2 Positive rates of acetylcholine provocation test across types of cancer in patients with active cancer

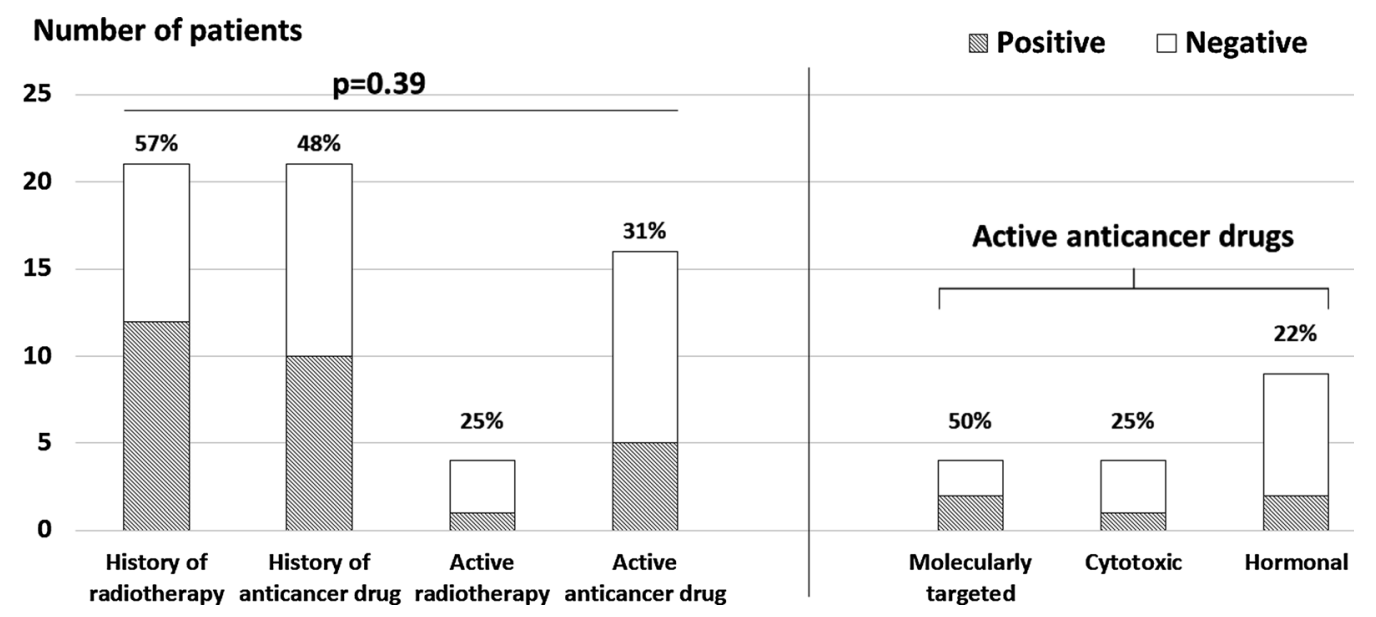

Fig. 3 Positive rates of acetylcholine test across types of anticancer treatment. Active cancer treatment is further divided into three groups (molecularly targeted, cytotoxic, and hormonal drugs)

seems biologically plausible that patients with cancer are likely to have coronary vasospasm. However, no study has addressed this issue.

\section{Vasospastic Angina in Cancer}

In a position paper from the European Society of Cardiology, chemotherapy including 5-FU and VEGF inhibitors and radiotherapy are listed as having effects on endothelial injury, 
Table 3 Factors associated with positive ACh provocation test

\begin{tabular}{|c|c|c|c|c|}
\hline \multirow[t]{2}{*}{ Variable } & \multicolumn{2}{|l|}{ Univariable } & \multicolumn{2}{|l|}{ Multivariable } \\
\hline & OR (95\% CI) & $p$ value & OR (95\% CI) & $p$ value \\
\hline Age (years) & $0.99(0.98-1.01)$ & 0.37 & $1.00(0.99-1.01)$ & 0.96 \\
\hline Men & $1.53(1.16-2.03)$ & 0.003 & $1.31(0.97-1.76)$ & 0.08 \\
\hline Body mass index $\left(\mathrm{kg} / \mathrm{m}^{2}\right)$ & $1.03(0.99-1.07)$ & 0.16 & $1.02(0.99-1.07)$ & 0.18 \\
\hline Hypertension & $0.87(0.65-1.15)$ & 0.33 & & \\
\hline Diabetes mellitus & $0.70(0.49-1.01)$ & 0.06 & $0.62(0.42-0.91)$ & 0.01 \\
\hline Dyslipidemia & $0.94(0.71-1.26)$ & 0.70 & & \\
\hline Current smoker & $1.95(1.32-2.86)$ & $<0.001$ & $1.74(1.15-2.64)$ & 0.009 \\
\hline Obstructive epicardial CAD & $1.54(1.12-2.13)$ & 0.008 & $1.56(1.11-2.18)$ & 0.01 \\
\hline Active cancer & $0.61(0.31-1.19)$ & 0.15 & $0.61(0.31-1.21)$ & 0.16 \\
\hline Active and a history of cancer & $1.03(0.70-1.52)$ & 0.88 & & \\
\hline Anticancer drug & $0.64(0.33-1.26)$ & 0.19 & & \\
\hline Radiotherapy & $1.04(0.47-2.31)$ & 0.92 & & \\
\hline
\end{tabular}

$A C h$ acetylcholine, $C A D$ coronary artery disease, $C I$ confidence interval, $O R$ odds ratio

vasospasm, and coronary atherosclerosis [12]. 5 -FU is used to treat patients with gastrointestinal and other malignancies and is reported to induce myocardial ischemia through vasospasm and endothelial injury in up to $10 \%$ of cases [12]. Indeed, patients treated with 5-FU experience chest pain in $1-18 \%[26,27]$. VEGF inhibitors are another class of drugs associated with angina at an incidence of $1-15 \%$. Inhibition of VEGF receptor impairs stimulation of endothelial nitric oxide activity, increases oxidative stress, and upregulates rho-kinase activity, contributing to coronary vasospasm [26]. In fact, several case reports have demonstrated coronary spasm induced by 5-FU and VEGF inhibitors [13-15]. However, it is difficult to determine in clinical practice whether chest pain in patients treated with anticancer drugs is induced by VSA. A recent retrospective singlecenter study demonstrated that 87 out of 4019 (2.2\%) patients treated with 5-FU were adjudicated as having VSA according to the presence of "typical chest pain" without any other diagnostic criteria [27]. In the present study, VSA was diagnosed by invasive intracoronary ACh provocation test, which has good diagnostic ability [16], and no significant relations between VSA and cancer or its treatment were shown. Even in patients actively treated with anticancer drugs, no impact was found with a positive ACh provocation rate of $31 \%$. Although patients who were receiving molecularly targeted drugs had a numerically higher rate of positive ACh test than those with cytotoxic and hormonal drugs $(50 \%$ vs. $25 \%$ vs. $22 \%$ ), all percentages were lower than the overall rate of positive ACh test in the present study $(51 \%)$. These findings may be robust because of consistent rates of positive ACh test across cancer types and multivariable analysis findings. Since patients with cancer often experience chest pain by numerous etiologies including myocardial ischemia and others [26], a pretest probability of having VSA might have been low in cancer patients in the present study. Further studies are needed to elucidate the impact of cancer and its treatment on coronary vasospasm. Beyond the relation between VSA and cancer, multivariable analysis showed current smoking and obstructive 
epicardial CAD as factors associated with coronary vasospasm in the present study. Smoking is a well-recognized risk factor for coronary spasm $[16,28]$, and a close relationship between coronary atherosclerosis and VSA has been shown in previous reports [29].

\section{Study Limitations}

The present study has several limitations. This was a single-center, retrospective, cross-sectional study. The overall sample size was not small, but subgroups of cancer and treatment had only small number of patients. Thus, larger, prospective studies are warranted. In patients with a history of cancer, data on the duration and dosage of chemotherapy and radiotherapy were not available. In addition, patient characteristics were different among the three groups. Even though a case report showed that intracoronary ACh provocation test confirmed the diagnosis of VSA in a patient with sorafenibinduced coronary artery spasm [14], whether ACh provocation test can identify patients with chemotherapy-induced coronary vasospasm as well as VSA patients who are not associated with anticancer therapies remains unclear.

\section{CONCLUSIONS}

Active and a history of cancer were not significantly associated with the diagnosis of VSA. We did not find an association of anticancer treatment including chemotherapy and radiotherapy with positive ACh provocation test in this cross-sectional observational study.

\section{ACKNOWLEDGEMENTS}

We thank the participants of the study.

Author Contributions. TM: writing, draft preparation, data gathering, formal analysis. YS: conceptualization, methodology, investigation, writing-review and editing, formal analysis. KS: investigation. KT: investigation. KK: conceptualization. HK: supervision. YK: project administration, writing-review and editing.

Funding. No funding or sponsorship was received for this study or publication of this article.

Authorship. All named authors meet the International Committee of Medical Journal Editors (ICMJE) criteria for authorship for this article, take responsibility for the integrity of the work as a whole, and have given their approval for this version to be published.

Disclosures. The authors (Tadahiro Matsumoto, Yuichi Saito, Kan Saito, Kazuya Tateishi, Ken Kato, Hideki Kitahara, and Yoshio Kobayashi) declare that they have nothing to disclose.

Compliance with Ethics Guidelines. The informed consent was obtained from all participants, and the study was reviewed and approved by the ethics committee of Chiba University Graduate School of Medicine. The study was conducted in accordance with this approval and adhered to the tenets of the Declaration of Helsinki revised in 2013.

Data Availability. The datasets generated during and/or analyzed during the current study are available from the corresponding author on reasonable request.

Open Access. This article is licensed under a Creative Commons Attribution-NonCommercial 4.0 International License, which permits any non-commercial use, sharing, adaptation, distribution and reproduction in any medium or format, as long as you give appropriate credit to the original author(s) and the source, provide a link to the Creative Commons licence, and indicate if changes were made. The images or other third party material in this article are included in the article's Creative Commons licence, unless indicated otherwise in a credit line to the material. If material is not included in the article's Creative Commons licence and your intended use is not permitted by statutory regulation or exceeds the permitted use, you 
will need to obtain permission directly from the copyright holder. To view a copy of this licence, visit http://creativecommons.org/licenses/by$\mathrm{nc} / 4.0 /$.

\section{REFERENCES}

1. Peter O, Ahmed A, Henrik SH, Eva P, Anastasios A, Udo S. Structural and functional coronary artery abnormalities in patients with vasospastic angina pectoris. Circ J. 2015;79:1431-8.

2. Kugiyama $K$, Yasue $H$, Okumura $K$, et al. Nitric oxide activity is deficient in spasm arteries of patients with coronary spastic angina. Circulation. 1996;94:266-71.

3. Ohyama K, Matsumoto Y, Takanami K, et al. Coronary adventitial and perivascular adipose tissue inflammation in patients with vasospastic angina. J Am Coll Cardiol. 2018;71:414-25.

4. Saito Y, Kitahara H, Shoji T, Nakayama T, Fujimoto Y, Kobayashi Y. Decreased double product at rest in patients with severe vasospasm. Heart Lung Circ. 2020;29:1511-6.

5. GBD 2013 Mortality and Causes of Death Collaborators. Global, regional, and national age-sex specific all-cause and cause-specific mortality for 240 causes of death, 1990-2013: a systematic analysis for the Global Burden of Disease Study 2013. Lancet 2015;385:117-71.

6. Koczwara B, Meng R, Miller MD, et al. Late mortality in people with cancer: a population-based Australian study. Med J Aust. 2021;214:318-23.

7. Sturgeon KM, Deng L, Bluethmann SM, et al. A population-based study of cardiovascular disease mortality risk in US cancer patients. Eur Heart J. 2019;40:3889-97.

8. Armenian $\mathrm{SH}, \mathrm{Xu} \mathrm{L}, \mathrm{Ky} \mathrm{B}$, et al. Cardiovascular disease among survivors of adult-onset cancer: a community-based retrospective cohort study. J Clin Oncol. 2016;34:1122-30.

9. Derek R, Lan P, Nanette CK, Gabor G, Edith P. Prevention of cardiovascular disease among cancer survivors: the role of pre-existing risk factors and cancer treatments. Curr Epidemiol Rep. 2017;4: 239-47.

10. Handy CE, Quispe R, Pinto X, et al. Synergistic opportunities in the interplay between cancer screening and cardiovascular disease risk assessment: together we are stronger. Circulation. 2018;138:727-34.

11. Okura Y, Ozaki K, Tanaka H, Takenouchi T, Sato N, Minamino T. The impending epidemic of cardiovascular diseases in patients with cancer in Japan. Circ J. 2019;83:2191-202.

12. Zamorano JL, Lancellotti $\mathrm{P}$, Rodriguez $\mathrm{MD}$, et al. 2016 ESC position paper on cancer treatments and cardiovascular toxicity developed under the auspices of the ESC Committee for Practice Guidelines: The Task Force for cancer treatments and cardiovascular toxicity of the European Society of Cardiology (ESC). Eur Heart J. 2016;37:2768-801.

13. Luwaert RJ, Descamps O, Majois F, Chaudron JM, Beauduin M. Coronary artery spasm induced by 5-fluorouracil. Eur Heart J. 1991;12:468-70.

14. Arima Y, Oshima S, Noda $\mathrm{K}$, et al. Sorafenib-induced acute myocardial infarction due to coronary artery spasm. J Cardiol. 2009;54:512-5.

15. Naib T, Steingart RM, Chen CL. Sorafenib-associated multivessel coronary artery vasospasm. Herz. 2011;36:348-51.

16. JCS Joint Working Group. Guidelines for diagnosis and treatment of patients with vasospastic angina (Coronary Spastic Angina) (JCS 2013). Circ J. 2014;78:2779-801.

17. Suzuki S, Kaikita K, Yamamoto E, Jinnouchi $H$, Tsujita K. Role of acetylcholine spasm provocation test as a pathophysiological assessment in nonobstructive coronary artery disease. Cardiovasc Interv Ther. 2021;36:52-3.

18. Tateishi K, Saito $\mathrm{Y}$, Kitahara $\mathrm{H}$, et al. Vasospastic angina and overlapping cardiac disorders in patients resuscitated from cardiac arrest. Heart Vessels. 2021;36:321-9.

19. Saito Y, Shoji T, Tateishi K, Kitahara H, Fujimoto Y, Kobayashi Y. Mental health status in patients undergoing intracoronary acetylcholine provocation test. Adv Ther. 2020;37:3807-15.

20. Saito Y, Kitahara H, Nishi T, Fujimoto Y, Kobayashi $Y$. Decreased resting coronary flow and impaired endothelial function in patients with vasospastic angina. Coron Artery Dis. 2019;30:291-6.

21. Tateishi K, Saito Y, Kitahara H, et al. Safety and usefulness of acetylcholine provocation test in patients with no culprit lesions on emergency coronary angiography. Int J Cardiol. 2018;269: 27-30. 
22. Saito Y, Kitahara H, Shoji T, et al. Relation between severity of myocardial bridge and vasospasm. Int J Cardiol. 2017;248:34-8.

23. Saito $\mathrm{Y}$, Kitahara $\mathrm{H}$, Shoji $\mathrm{T}$, et al. Intracoronary acetylcholine provocation testing-omission of the $20-\mu \mathrm{g}$ dose is feasible in patients without coronary artery spasm in the other coronary artery. Circ J. 2016;80:1820-3.

24. Sueda S, Kohno H. Transitional changes of acetylcholine spasm provocation test procedures. Cardiovasc Interv Ther. 2020;35:321-6.

25. Bharadwaj A, Potts J, Mohamed MO, et al. Acute myocardial infarction treatments and outcomes in 6.5 million patients with a current or historical diagnosis of cancer in the USA. Eur Heart J. 2020;41: 2183-93.
26. Herrmann J, Yang EH, Iliescu CA, et al. Vascular toxicities of cancer therapies: the old and the newan evolving avenue. Circulation. 2016;133: 1272-89.

27. Zafar A, Drobni ZD, Mosarla R, et al. The incidence, risk factors, and outcomes with 5-fluorouracil-associated coronary vasospasm. JACC CardioOncol. 2021;3:101-9.

28. Morrow JD, Frei B, Longmire AW, et al. Increase in circulating products of lipid peroxidation (F2-isoprostanes) in smokers. Smoking as a cause of oxidative damage. N Engl J Med. 1995;332: 1198-203.

29. Tsujita K, Sakamoto K, Kojima S, et al. Coronary plaque component in patients with vasospastic angina: a virtual histology intravascular ultrasound study. Int J Cardiol. 2013;168:2411-5. 